\title{
Glycogen and Trehalose Accumulation during Colony Development in Streptomyces antibioticus
}

\author{
By ALFREDO F. BRAÑA, CARMEN MÉNDEZ, LUIS A. DÍAZ, \\ MANUEL B. MANZANAL AND CARLOS HARDISSON* \\ Departamento de Microbiologia, Facultad de Medicina, Universidad de Oviedo, 33006 Oviedo, \\ Spain
}

(Received 16 July 1985)

\begin{abstract}
Streptomyces antibioticus accumulated glycogen and trehalose in a characteristic way during growth on solid medium. Glycogen storage in the substrate mycelium took place during development of the aerial mycelium. The concentration of nitrogen source in the culture medium influenced the time at which accumulation started as well as the maximum levels of polysaccharide stored. Degradation of these glycogen reserves was observed near the beginning of sporulation. The onset of sporogenesis was always accompanied by a new accumulation of glycogen in sporulating hyphae. During spore maturation the accumulated polysaccharide was degraded. No glycogen was observed in aerial non-sporulating hyphae or in mature spores. Trehalose was detected during all phases of colony development. A preferential accumulation was found in aerial hyphae and spores, where it reached levels up to $12 \%$ of the cell dry weight. The possible roles of both carbohydrates in the developmental cycle of Streptomyces are discussed.
\end{abstract}

\section{INTRODUCTION}

The morphological changes that take place during growth and differentiation of streptomycete colonies are relatively well known due to several electron microscopic studies (Wildermuth, 1970; Mendez et al., 1985a). However, less information is available on the physiological events that occur during aerial mycelium growth and sporogenesis. This may, in part, be due to the technical difficulties imposed by the fact that these differentiation processes take place only on solid media.

In a previous paper (Braña et al., 1982) we described the ability of Streptomyces antibioticus to accumulate glycogen as a reserve compound. We have also detected the presence of trehalose in the spores and mycelia of this micro-organism. Storage compounds such as glycogen and trehalose have been frequently associated with biochemical differentiation in micro-organisms (Dawes \& Senior, 1973; Thevelein, 1984). In the present work, chemical and electron microscopic techniques were used to study the changes which the reserve compounds undergo in the course of the developmental cycle of $S$. antibioticus.

\section{METHODS}

Micro-organism and culture conditions. Streptomyces antibioticus ATCC 11891 was grown as lawns on sterile cellophane films placed on solid glucose/asparagine/yeast extract medium (GAE medium; Hardisson \& Manzanal, 1976). The plates (containing $30 \mathrm{ml}$ culture medium) were inoculated by spreading $0.2 \mathrm{ml}$ of a spore suspension, obtained as described previously (Hardisson et al., 1978), on the surface of the cellophane and were incubated at $28^{\circ} \mathrm{C}$. The developmental stage of colonial growth was followed by observing the changes in coloration of the surface of the cultures, and by light microscopic observation of semi-thin sections of the colonies (Mendez et al., 1985a).

Analytical procedures. All determinations were done independently with three plates for every sampling time. At various times of incubation, the cellophane membranes bearing the bacterial mass were removed from the plates. 
Glucose and nitrogen source content in the culture medium were determined as follows. One agar plug (14 $\mathrm{mm}$ in diameter) was cut out with a cork borer from the centre of each plate, fragmented into small pieces and suspended in $1 \mathrm{ml}$ distilled water. After heating to $100^{\circ} \mathrm{C}$ for $10 \mathrm{~min}$, water was added to obtain a final volume of $10 \mathrm{ml}$. The glucose present in the solutions obtained was measured by the glucose oxidase-peroxidase method (Lloyd $\&$ Whelan, 1969). Ninhydrin-positive compounds were determined by the procedure of Rosen (1957), to estimate the content of nitrogen sources in the culture medium.

For glycogen determinations, the mycelium grown on each plate was gently scraped from the cellophane and suspended in $5 \mathrm{ml}$ distilled water. The suspensions were heated to $100^{\circ} \mathrm{C}$ for $5 \mathrm{~min}$ and then disrupted with glass beads $\left(0.1 \mathrm{~mm}\right.$ diameter) in a Vibrogen homogenizer at $0^{\circ} \mathrm{C}$ for $15 \mathrm{~min}$. After removal of the beads by decantation, the glycogen content of the samples was assayed with amyloglucosidase (Sigma), as previously described (Braña $e t$ al., 1982).

For trehalose measurements, TCA $(0.5 \mathrm{~m}$ final concentration) was added to samples of the disrupted suspensions. The samples were extracted overnight at $4{ }^{\circ} \mathrm{C}$ with shaking. After centrifugation, the trehalose content in the supernatants was estimated by the anthrone method. Glucose was used as standard and the values obtained were expressed as glucose equivalents. Trehalose was the only soluble carbohydrate detected after treatment with $0.5 \mathrm{M}$-TCA, as confirmed by descending paper chromatography on Whatman no. 1 filter paper using 1-propanol/ethyl acetate/water $(7: 1: 2$, by vol.) as solvent. Trehalose in the chromatograms was detected with the alkaline silver nitrate reagent of Trevelyan et al. (1950).

For dry cell weight determinations, samples $(1.5 \mathrm{ml})$ of disrupted mycelial suspensions were collected in preweighed glass vials and dried at $100{ }^{\circ} \mathrm{C}$ to constant weight.

Electron microscopy. At various times of growth, samples of mycelium were removed from the cellophane, fixed in $1 \%(\mathrm{w} / \mathrm{v}$ ) osmium tetroxide (Ryter \& Kellenberger, 1958), dehydrated in acetone and embedded in Epon 812 . To observe glycogen, pale gold ultrathin sections were treated with periodic acid, thiocarbohydrazide and silver proteinate, according to Thiery (1967). Photographs of sections were taken with a Philips EM-300 electron microscope at $60 \mathrm{kV}$.

\section{RESULTS}

\section{Changes in carbohydrate content during colony growth}

The glycogen and trehalose contents of the mycelium during colony differentiation in $S$. antibioticus were measured. Residual glucose and nitrogen sources in the culture medium were also determined (Fig. 1). Glycogen was detected after about $32 \mathrm{~h}$ of growth. At this time, only $7 \%$ of the initial ninhydrin-positive compounds were detected in the culture medium, whereas the glucose concentration was about $50 \%$ of the initial value. During growth of the aerial mycelium, glycogen accumulation continued, reaching a maximum $(18 \%$ of the total cell dry weight) just before the onset of the sporulation phase (at about $62 \mathrm{~h}$ of incubation). Subsequently, the glycogen content of the mycelium decreased gradually.

A new experiment was designed to verify the remarkable coincidence between the time of nitrogen exhaustion, the formation of aerial mycelium and the beginning of glycogen accumulation. When the asparagine concentration of the medium was lowered to $0.25 \%$ (Fig. $2 a$ ), formation of aerial mycelium and accumulation of glycogen occurred earlier than in the control culture containing $1 \%$ asparagine (Fig. 1). Conversely, in the presence of a higher asparagine concentration $(2 \%$; Fig. $2 b)$ the appearance of aerial mycelium and glycogen was delayed. In every case, glycogen synthesis and aerial mycelium formation seemed to be linked to the exhaustion of the ninhydrin-positive compounds in the medium. Maximum levels of glycogen were also influenced by the nitrogen content of the medium, being highest at $0.25 \%$ asparagine and lowest at $2 \%$ asparagine.

The pattern of trehalose accumulation during colony development was different from that of glycogen. Trehalose was detected in the substrate hyphae from the initial stages of growth, and its levels remained constant during the first $28 \mathrm{~h}$ of substrate growth (Fig. 1). Synthesis of trehalose, unlike that of glycogen, took place in the presence of the available nitrogen source. During growth of the aerial mycelium, total trehalose content of the colony increased from $3 \%$ to $7 \%$ of the dry cell weight, remaining at this level throughout the sporulation process.

\section{Cytochemical studies}

Ultrathin sections of colonies at various times of growth were stained with silver proteinate to demonstrate glycogen and observed in the electron microscope. After $24 \mathrm{~h}$ incubation (Fig. $3 a$ ), 

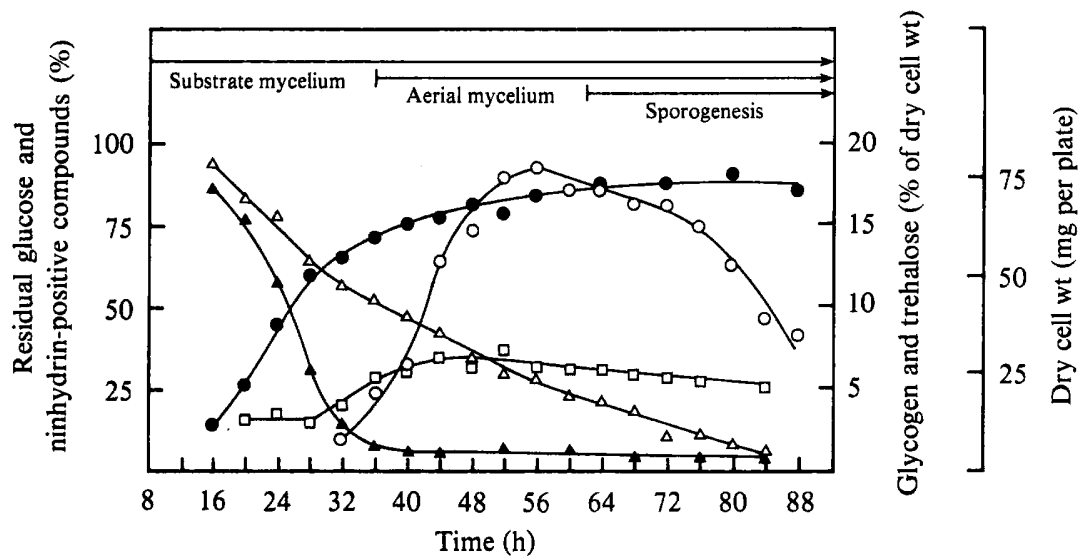

Fig. 1. Growth, sugar accumulation and nutrient depletion during colony development of $S$. antibioticus on solid GAE medium covered with a cellophane membrane. The mycelium was removed from the plates and dry cell weight $(O)$ and the accumulated glycogen $(O)$ and trehalose $(\square)$ were determined. Residual glucose $(\triangle)$ and ninhydrin-positive compounds $(\Delta)$ were measured in samples of medium previously homogenized in distilled water. The different phases of colony development are indicated at the top of the figure. The results are means of three determinations.

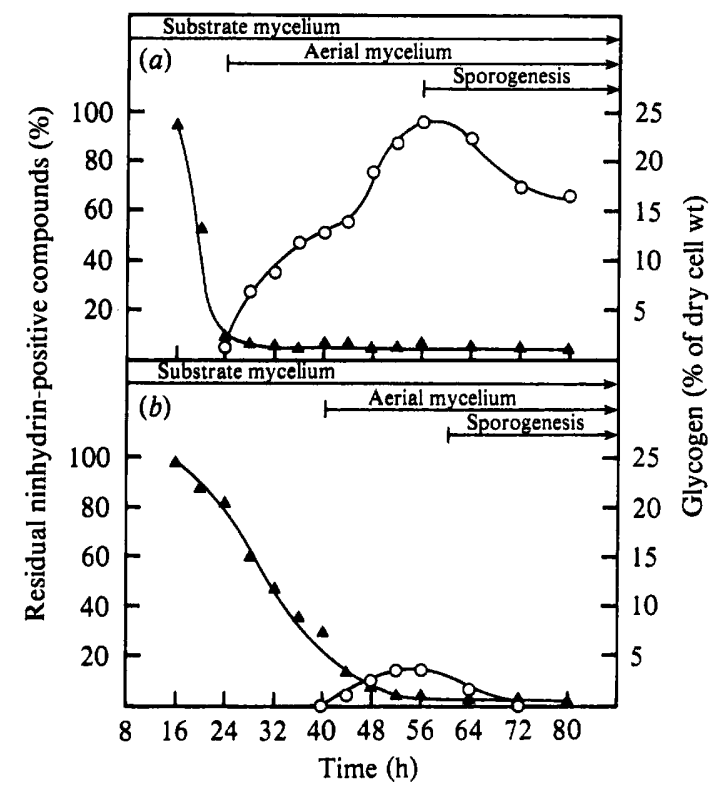

Fig. 2. Residual ninhydrin-positive compounds $(\boldsymbol{A})$ and accumulation of glycogen $(O)$ in media with $0.25 \%$ asparagine $(a)$ or $2 \%$ asparagine $(b)$.

the colonies contained only substrate mycelium and no polysaccharide granules were present in the hyphae. After about $36 \mathrm{~h}$ growth, two different regions could be distinguished (Fig. $3 \mathrm{~b}$ ). The lower region contained a large and dense network of substrate hyphae, whereas in the upper region a loose network of aerial hyphae had started to form. In the substrate region many cells appeared completely filled with glycogen granules (Fig. $3 c$ ). In the basal zone of the colony, substrate hyphae were lysed and devoid of glycogen (Fig. $3 d$ ). In a $48 \mathrm{~h}$ colony, a well-developed aerial mycelium could be observed (Fig. $4 a$ ). At this time of colony development many substrate 

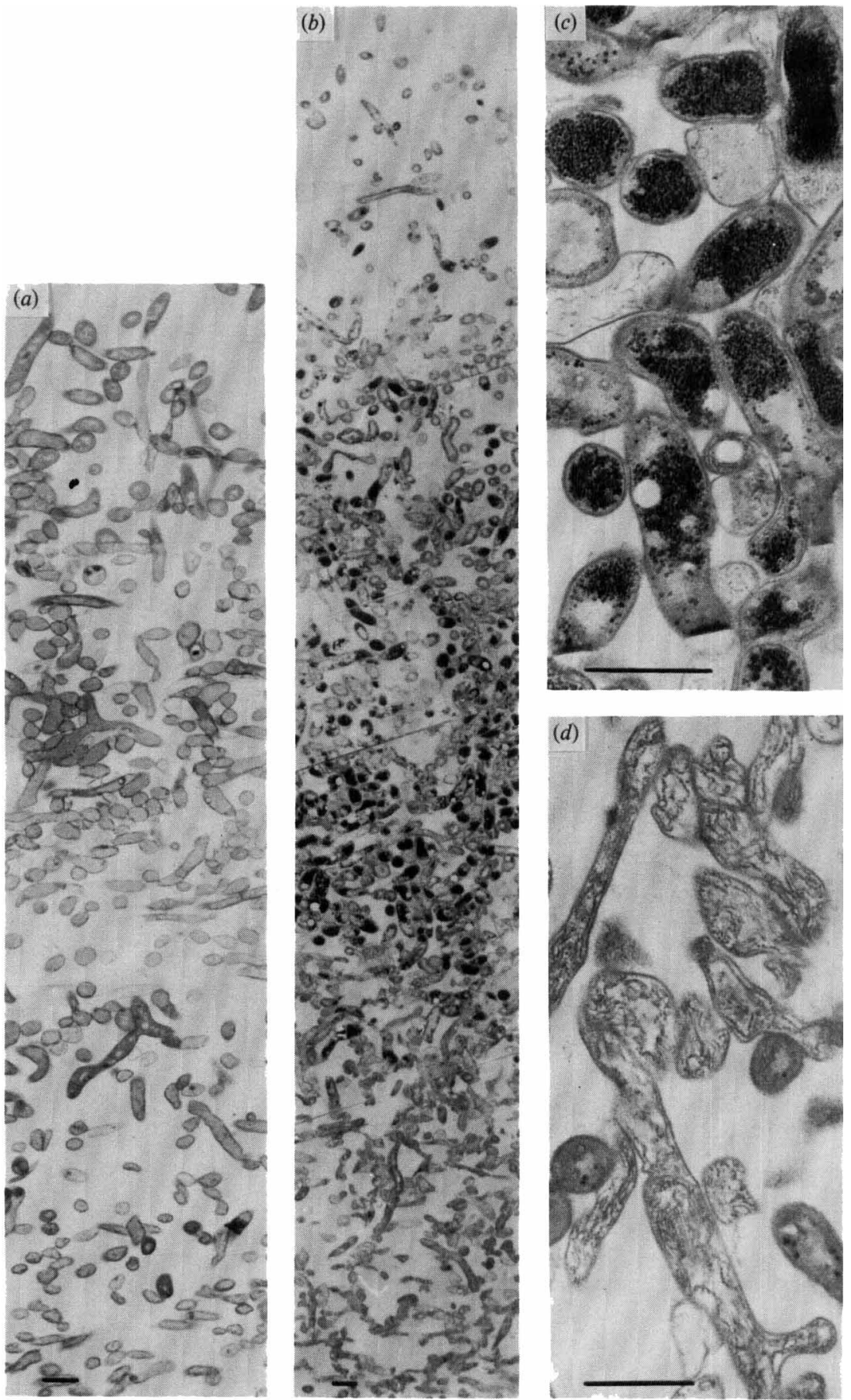

Figs 3-5. Thin sections of $S$. antibioticus colonies stained specifically to demonstrate polysaccharides.

Fig. 3. Vertical sections of colonies grown for $24 \mathrm{~h}(a)$ or $36 \mathrm{~h}(b-d)$. After $24 \mathrm{~h}$ of incubation the entire colony contained only substrate hyphae and no glycogen granules were observed $(a)$. In colonies at $36 \mathrm{~h}$ incubation a loose network of aerial hyphae appeared in the upper region $(b)$. In the central region of the colony many substrate hyphae contained glycogen granules $(c)$, whereas in the basal zone the substrate hyphae were mostly lysed and lacked glycogen $(d)$. Bars, $1 \mu \mathrm{m}$. 
Glycogen and trehalose in S. antibioticus
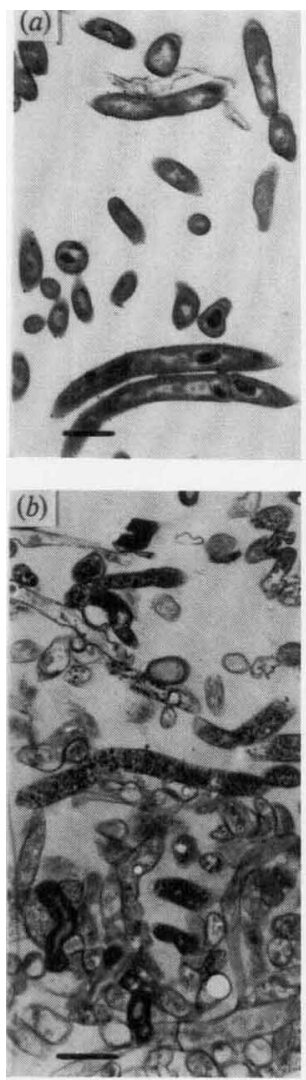
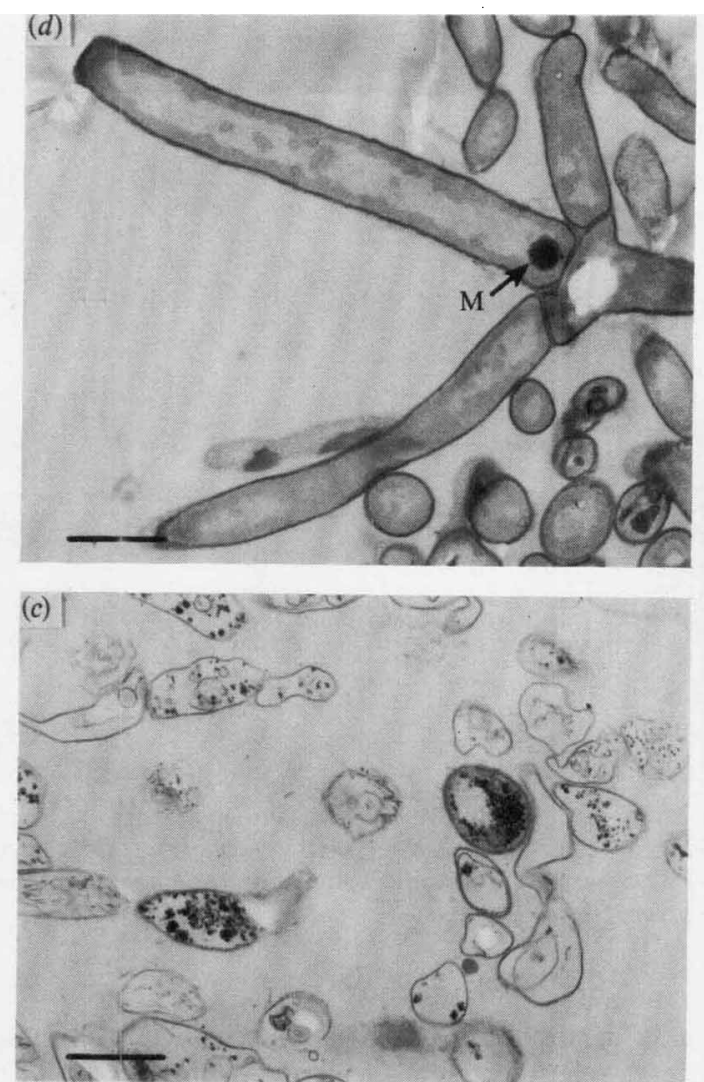
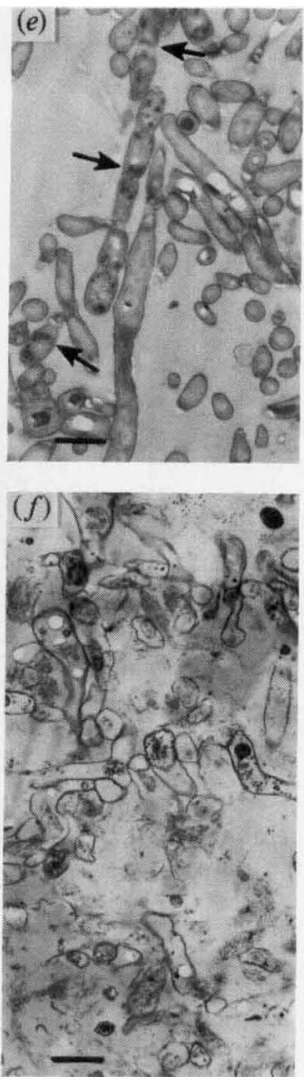

Fig. 4. Vertical sections of colonies grown for $48 \mathrm{~h}(a-c)$ or $72 \mathrm{~h}(d-f)$, showing aerial $(a, d, e)$ and substrate $(b, c, f$ mycelium. In the substrate mycelium of the $48 \mathrm{~h}$ colony many lysed cells with some glycogen granules were visible $(c)$. In the $72 \mathrm{~h}$ sporulating colony, the cytoplasm of aerial hyphae $(d)$ contained no glycogen granules, and only the mesosomes $(\mathbf{M})$ were intensely stained. In the upper region of the aerial mycelium (e) some sporulating hyphae could be observed (arrows). The cytoplasm of sporulating hyphae contained glycogen granules (also see Fig. 5). The substrate mycelium at this stage (f) was completely lysed. Bars, $1 \mu \mathrm{m}$.

hyphae were lysed but still retained part of their glycogen content among the cell debris (Fig. $4 b, c)$.

In sporulating colonies (after $72 \mathrm{~h}$ of incubation) the substrate mycelium was completely lysed, although glycogen granules were still visible (Fig. $4 f$ ). In the aerial region (Fig. $4 e$ ) a new round of glycogen synthesis could be observed. It is important to note that in this region and at this time of colony development, glycogen synthesis took place only in sporulating hyphae (Fig. $4 e$, Fig. 5), and in no case were glycogen granules seen in the cytoplasm of non-sporulating aerial hyphae (Fig. $4 d$ ). The sequential development of glycogen during the sporulation process is shown in Fig. 5. The synthesis of glycogen coincided with the formation of the sporulation septum (Fig. $5 a$ ) and the maximum polysaccharide accumulation was reached during the first stages of maturation (Fig. $5 b$ ). Later, the number of granules progressively decreased and in mature spores no glycogen granules were observed (Fig. $5 c$ ). Chemical analysis of isolated spores did not reveal the presence of glycogen.

\section{Trehalose content in hyphae and spores}

To obtain substrate hyphae, cultures were grown on cellophane membranes and the mycelium was collected from $16 \mathrm{~h}$ cultures where the aerial hyphae had not yet formed. However, to obtain suspensions with only aerial hyphae or spores, the micro-organism was grown directly on 

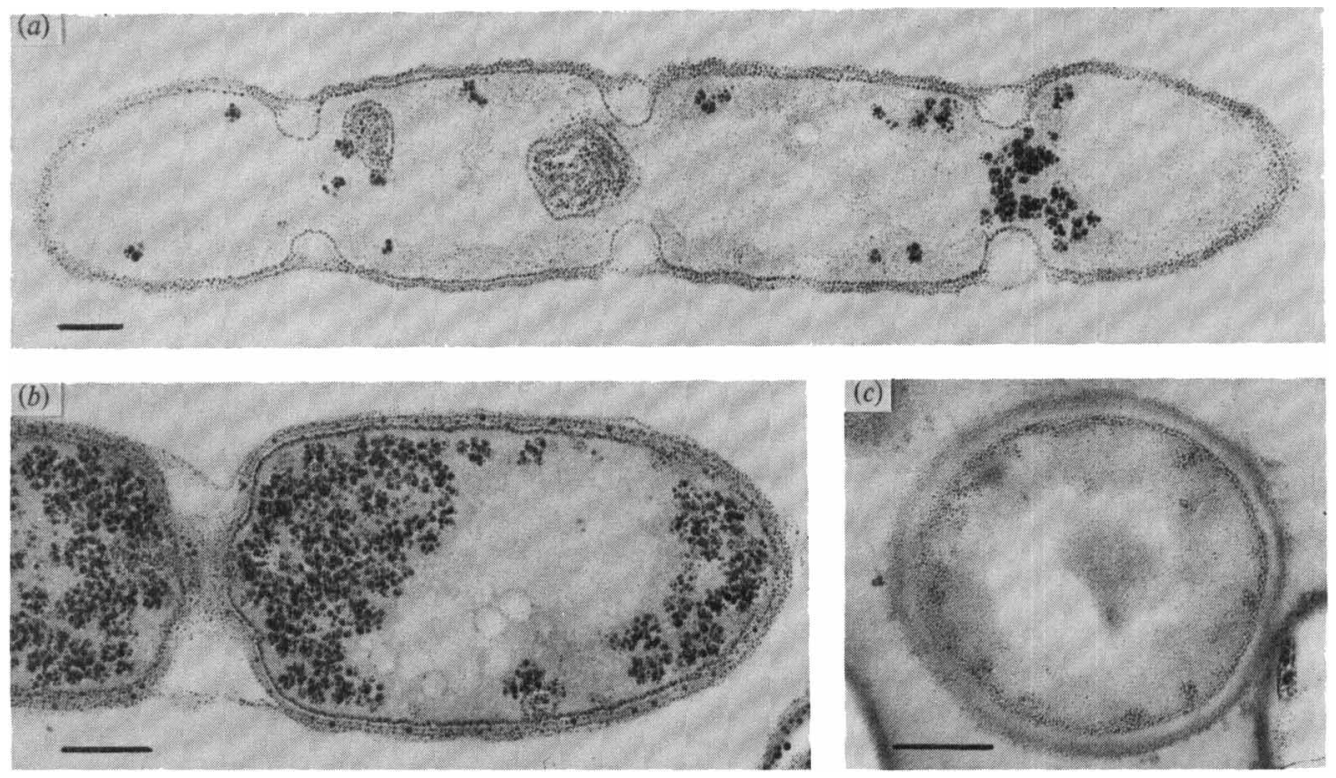

Fig. 5. Sequential development of glycogen granules during sporogenesis. The appearance of glycogen granules coincided with the formation of the sporulation septa $(a)$. Glycogen content increased until maturation began $(b)$. During maturation, the number of granules progressively decreased and in mature spores $(c)$ no glycogen granules were observed. Bars, $200 \mathrm{~nm}$.

solid GAE medium. Under these conditions most of the substrate hyphae grew into the medium, which facilitated their detachment from the aerial mycelium. Aerial hyphae were obtained from $48 \mathrm{~h}$ cultures by carefully scraping the surface of the colony with a spatula. Spores were harvested from cultures after $7 \mathrm{~d}$ incubation using glass beads as reported previously (Hardisson et al., 1978). Trehalose content (expressed as a percentage of dry cell weight) in the different types of cells was $2 \%$ for substrate hyphae, $5 \%$ for aerial hyphae and $12 \%$ for spores.

\section{DISCUSSION}

The occurrence, accumulation and utilization of glycogen and trehalose followed different patterns during the developmental cycle of $S$. antibioticus. This different behaviour suggests that these carbohydrates may play distinct physiological roles during colony differentiation.

Accumulation of glycogen in the substrate mycelium may be triggered by the exhaustion of the nitrogen source, as observed in many micro-organisms (Dawes \& Senior, 1973). Several observations, however, suggest that part, if not all, of the glycogen synthesized by the substrate hyphae is not utilized by these cells. Despite the lysis of many substrate hyphae containing glycogen, the total glycogen content of the colony increased steadily during growth of the aerial hyphae (which at this time of colony development did not accumulate glycogen). In this period, more than $25 \%$ of the initial glucose was still present in the culture medium, which seems to make glycogen degradation unnecessary as a source of carbon and energy. In addition, many polysaccharide granules were visible among the cell debris in the substrate mycelium. This seems to indicate that substrate hyphae death was not due to a shortage of carbon source as their reserves remained unused after lysis. A clear decrease in glycogen levels of the colony only occurred during the sporulation phase when glucose in the medium approached exhaustion.

The temporal relationship between the exhaustion of the nitrogen source and the onset of aerial mycelium formation is also interesting. It is well known that differentiation of aerial mycelium in Streptomyces is affected by the nitrogen sources in the culture medium. Complex nitrogen sources such as meat extract (Kalakoutski \& Agre, 1976) or casein hydrolysate 
(Coleman \& Ensign, 1982) often suppress differentiation. Our results using different asparagine concentrations suggest that nitrogen exhaustion in the medium may be one of the factors involved in triggering aerial mycelium formation. On the other hand, growth of aerial hyphae without a supply of nitrogen source from the medium can probably be explained by the utilization of the substrate mycelium as a nutrient support (Mendez et al., 1985b). Thus, two well-defined microenvironments are established within the colony: in the lower region the substrate hyphae accumulate glycogen and lyse, whereas growth of differentiated hyphae continues in the upper region.

In the aerial hyphae, glycogen synthesis and sporulation appeared to be related because: (i) glycogen was only detected in sporulating aerial hyphae and (ii) sporulating hyphae lacking glycogen granules were not observed. The glycogen accumulated during sporogenesis was absent in mature spores, which clearly excludes any role for this polysaccharide as a reserve compound for the germination process. On the other hand, the gradual disappearance of glycogen during maturation suggests its involvement in the synthesis of some spore components or as a source of energy to complete the sporulation process, as has been proposed for other sporulating microorganisms (Bergere et al., 1975; Braña et al., 1980; Kane \& Roth, 1974; Strasdine, 1972).

Accumulation of trehalose seems to be widespread among Streptomyces species (Elbein, 1967; Ensign, 1982; Nimi et al., 1984), which suggests a significant role of this disaccharide in the biology of these micro-organisms. In S. antibioticus trehalose was present in all phases of colony development and did not seem to behave as a typical storage carbohydrate. In addition its localization differed from that of glycogen, with a greater accumulation in the aerial mycelium and, especially, in the spores. Trehalose is known to protect the structural and functional integrity of isolated membranes under conditions of desiccation (Crowe et al., 1984). Taking into account the preferential accumulation of trehalose in the regions of the colony farthest from the humidity of the culture medium, it is quite tempting to propose an involvement of this compound in protecting the aerial mycelium and/or the spores from desiccation. We have, in fact, found evidence of such an effect with exogenous trehalose added to mycelium and spores (unpublished results). This mechanism might be particularly important for bacteria such as Streptomyces spp., which possess the remarkable ability of growing and sporulating in the air. Other possibilities concerning the high levels of trehalose accumulated in the spores must be borne in mind: (i) trehalose may be used to initiate the growth cycle, supplying the energy necessary for the initial stages of germination, as has previously been proposed for Streptomyces hygroscopicus (Hey-Ferguson et al., 1973) and several fungi (Thevelein, 1984); (ii) this sugar may be used as an endogenous source of energy during the dormant period (Thevelein, 1984; Barton et al., 1982). At present, none of the above-mentioned possible functions can be excluded and further studies are required to determine the exact role of trehalose during the life cycle of Streptomyces spp.

C.M. was the recipient of a predoctoral fellowship from the Ministry of Education of Spain.

\section{REFERENCES}

Barton, J. K., Den Hollander, J. A., Hopfield, J. J. \& ShulmaN, R. G. (1982). ${ }^{13} \mathrm{C}$ nuclear magnetic resonance study of trehalose mobilization in yeast spores. Journal of Bacteriology 151, 177-185.

Bergere, J. L., Rousseau, M. \& Mercier, C. (1975). Polyoside intracellulaire impliqué dans la sporulation de Clostridium butyricum. Annales de microbiologie 126A, 295-314.

Braña, A. F., Manzanal, M. B. \& Hardisson, C. (1980). Occurrence of polysaccharide granules in sporulating hyphae of Streptomyces viridochromogenes. Journal of Bacteriology 144, 1139-1142.

Braña, A. F., Manzanal, M. B. \& Hardisson, C. (1982). Characterization of intracellular polysac- charides of Streptomyces. Canadian Journal of Microbiology 28, 1320-1323.

Coleman, R. H. \& ENSIGN, J. C. (1982). Regulation of formation of aerial mycelia and spores of Streptomyces viridochromogenes. Journal of Bacteriology 149, 1102-1111.

Crowe, L. W., Mouradian, R., Crowe, J. H., JACKSON, S. A. \& WOMERSLEY, C. (1984). Effects of carbohydrates on membrane stability at low water activities. Biochimica et biophysica acta 769, 141150.

DAWES, E. A. \& SENIOR, P. J. (1973). Energy reserve polymers in micro-organisms. Advances in Microbial Phisiology 10, 136-266. 
ElbeIN, A. D. (1967). Carbohydrate metabolism in streptomycetes. II. Isolation and enzymatic synthesis of trehalose. Journal of Bacteriology 94, 15201524.

ENSIGN, J. C. (1982). Developmental biology of actinomycetes. In Overproduction of Microbial Products, pp. 127-140. Edited by V. Krumphanzl, B. Sikyta \& Z. Vanek. London \& New York: Academic Press.

Hardisson, C. \& Manzanal, M. B. (1976). Ultrastructural studies of sporulation in Streptomyces. Journal of Bacteriology 127, 1443-1454.

Hardisson, C., Manzanal, M. B., Salas, J. A. \& SuAREZ, J. E. (1978). Fine structure, physiology and biochemistry of arthrospore germination in Streptomyces antibioticus. Journal of General Microbiology 105, 203-214.

Hey-Ferguson, A., Mitchell, M. \& Elbein, A. D. (1973). Trehalose metabolism in germinating spores of Streptomyces hygroscopicus. Journal of Bacteriology 116, 1084-1085.

KalaKoutskir, L. V. \& AGRE, N. S. (1976). Comparative aspects of development and differentiation in actinomycetes. Bacteriological Reviews 40, 469524.

KANE, S. M. \& Roth, R. (1974). Carbohydrate metabolism during ascospore development in yeast. Journal of Bacteriology 118, 8-14.

LLOYD, J. B. \& WhelaN, W. J. (1969). An improved method for enzymic determination of glucose in the presence of maltose. Analytical Biochemistry 30, 467470.

Mendez, C., Barbes, C., Manzanal, M. B. \& HARDisson, C. (1985a). A simple method to study colony development in Streptomyces. FEMS Microbiology Letters 28, 99-102.

Mendez, C., Braña, A. F., Manzanal, M. B. \& HARDISSON, C. $(1985 b)$. Role of substrate mycelium in colony development in Streptomyces. Canadian Journal of Microbiology 31, 446-450.

Nimi, O., Amano, Y., IKeda, A., Yoshimura, Y., SugIYAMA M. \& NoMI, R. (1984). Intracellular accumulation of trehalose during streptomycin formation by Streptomyces griseus. Agricultural and Biological Chemistry 48, 285-290.

Rosen, H. (1957). A modified ninhydrin colorimetric analysis for amino acids. Archives of Biochemistry and Biophysics 67, 10-15.

RYTER, A. \& KellenBerger, E. (1958). Etude au microscope électronique de plasma contenant de l'acide désoxyribonucléique. Zeitschrift für Naturforschung 13b, 597-605.

Strasdine, G. A. (1972). The role of intracellular glucan in endogenous fermentation and spore maturation in Clostridium botulinum type E. Canadian Journal of Microbiology 18, 211-217.

ThevelerN, J. M. (1984). Regulation of trehalose mobilization in fungi. Microbiological Reviews 48, 42-59.

THIERY, J. P. (1967). Mise en évidence des polysaccharides sur coupes fines en microscopie électronique. Journal de Microscopie 6, 987-1018.

Trevelyan, W. E., Procter, D. P. \& Harrison, J. S. (1950). Detection of sugars on paper chromatograms. Nature, London 166, 444-445.

WILDERMUTH, H. (1970). Development and organization of the aerial mycelium in Streptomyces coelicolor. Journal of General Microbiology 60, 43-50. 